\title{
Balancing shape distortions and contiguity in cartograms
}

\author{
Michael T. Gastner ${ }^{\mathrm{a}}$, Simon T. Perrault ${ }^{\mathrm{b}}$, Chen-Chieh Feng ${ }^{\mathrm{c}}$ \\ ${ }^{a}$ Yale-NUS College, Singapore 138527, Singapore-michael.gastner@yale-nus.edu.sg \\ ${ }^{b}$ Singapore University of Technology and Design, Singapore 487372, Singapore - simon_perrault@sutd.edu.sg \\ ${ }^{c}$ Department of Geography, National University of Singapore, Singapore 117570, Singapore - chenchieh.feng@nus.edu.sg \\ * Corresponding author
}

Keywords: cartogram, map design, map projections, usability issues

\begin{abstract}
:
Cartograms are thematic maps in which areas (e.g., countries or provinces) are rescaled in proportion to a quantitative variable (e.g., population size or gross domestic product). Ideally, cartograms should correctly represent which geographic regions are neighbors while also preserving the shapes of the regions. For realistic data, it is impossible to simultaneously satisfy both objectives: if neighboring regions are rescaled and forced to be connected along shared boundaries, it is necessary to distort their shapes. For example, let us consider the distribution of seasonal influenza vaccine doses in Africa in 2013 (data from Palache et al. 2015). In Figure 11a), we visualize the data with a choropleth map using a conventional equal-area projection. Following recommended practices (Foster, 2019), colors on this choropleth map represent normalized data (doses per 1000 inhabitants). Because of normalization, the number of doses available in each country cannot be inferred directly from Figure 1 (a).

Cartograms offer a way to represent non-normalized data. The cartogram in Figure 1 (b) shows an example in which countries are scaled by the number of doses. This example is based on a density-equalizing projection constructed using the flow-based algorithm by Gastner et al. (2018). The flow-based algorithm guarantees that the cartogram is contiguous. That is, all neighbors in conventional geographic space are neighbors in the cartogram and vice versa. Moreover, every point in Figure 1 b) can be uniquely projected back to a point on the equal-area map in Figure 1 1 a). This property is mathematically desirable, but it comes at the cost of readability. Because the distribution of vaccine doses is strongly skewed towards South Africa and some countries in North Africa, the cartogram in Figure 1.b) is so distorted that countries in Central Africa are difficult to identify by their shapes. In general, whenever the mapping variable is concentrated in only a few regions, a density-equalizing projection inevitably generates severe distortions.
\end{abstract}

When the mapping variable is spatially heterogeneous, we suggest that it is better to relax contiguity as shown in Figure 1(c). In this cartogram, countries with fewer than 20,000 doses are represented by small circular nodes, and contiguity is represented by thin gray lines. This cartogram design has the following benefits:

- There is enough space to label even those countries in Figure 11 (c) that receive very few doses. Identifying regions with small values provides valuable insights into the data.

- The contiguity graph in Figure 1(c), formed by the small circular nodes and gray lines, represents the neighborhood relationships in a more legible manner than the cluttered center of Figure 1 (b).

- By placing each circle at the approximate location of the country's centroid, the overall shape of Africa is more recognizable in Figure 11(c) than in Figure 1 b).

Countries with more than 20,000 doses are still represented as polygons in Figure 1.(c), and all neighboring polygons remain connected. For example, the North African countries from Morocco in the west to Egypt in the east are shown as a contiguous sub-cartogram generated by a density-equalizing projection. The distortions in this sub-cartogram are mild, so a reader can easily identify these countries when comparing the cartogram to the equal-area map in Figure 1(a). We recommend that density-equalizing projections should be applied at least to some parts of the map because the resulting visualization contains more cartographic details (e.g., whether three countries share a tripoint border) than the contiguity graph (which only reveals pairwise contiguity).

Previous classifications of cartogram designs distinguished between contiguous and non-contiguous cartograms. In the latter case, gaps are deliberately inserted between all regions so that shapes can be maintained perfectly (Olson, 1976). A disadvantage of non-contiguous cartograms is that they do not attempt to indicate neighborhood relationships. In our opinion, inserting thin lines to indicate the contiguity graph, as demonstrated in Figure 1. (c), is visually unobtrusive and should therefore be regularly included in future cartogram designs. 


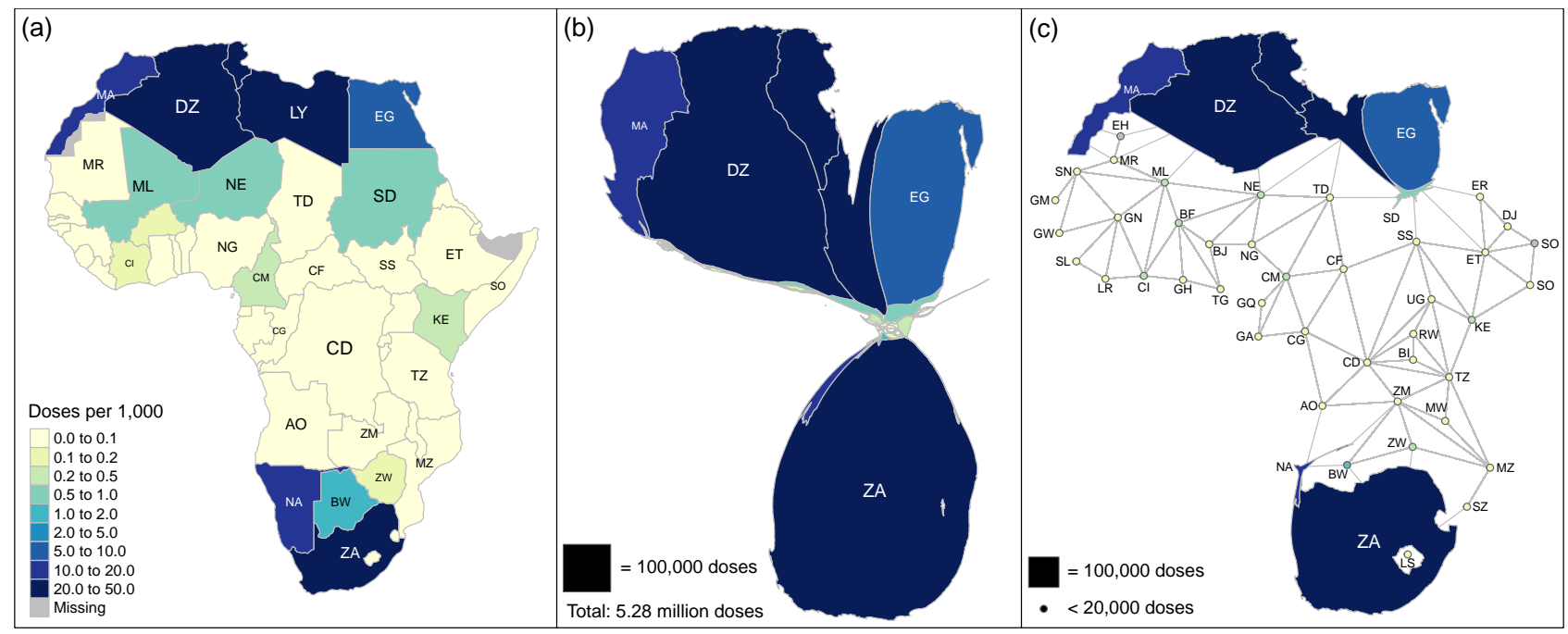

Figure 1. Distribution of seasonal influenza vaccines in Africa in 2013.

Our proposed design is reminiscent of Dorling's (1996) circular cartograms. However, there are two important differences. First, Dorling cartograms represent every region with a circle, whereas Figure 1(c) only uses circles where the mapping variable is below a fixed, small threshold. Second, the circles in Dorling cartograms have an area in proportion to the mapping variable, whereas we do not vary the circle area so that the colors inside the circles (representing normalized data) remain discernible even if the non-normalized (i.e., total) number is small. We also note that Dorling cartograms are not guaranteed to be contiguous: circles in Dorling cartograms may touch each other even if the corresponding regions are not adjacent, and, conversely, not every pair of neighboring regions is necessarily depicted by touching circles. A compromise between Dorling's and our design would be to represent each region by a circle and scale each circle according to the mapping variable, but deliberately insert gaps between all circles so that it is possible to indicate the contiguity graph with thin lines between the circles.

The example shown in Figure 1 (c) was constructed by hand as a "proof of concept." We chose a threshold of 20,000 doses because our subjective impression is that it results in a reasonable balance between shape distortion and contiguity. In the future, we will employ an objective criterion based on a quantitative measure of shape distortion (e.g., the turning function similarity defined by Arkin et al. 1991). We will experimentally determine to what extent users can tolerate shape distortion before the cartogram becomes too difficult to read. To reduce the time and effort required to construct contiguous cartograms with limited shape distortion, we will develop an algorithm that automates the placement of nodes in the contiguity graph. We intend to integrate this algorithm into the web application https://go-cart.io//Tingsheng et al. 2019). With a web-based implementation, we hope that it will be easy for cartographers to create cartograms that are consistent with the proposed design principles.

\section{Acknowledgements}

This work was supported by the Singapore Ministry of Education (AcRF Tier 1 Grant IG18-PRB104, R-607-000-401114). We would like to thank Editage (www.editage.com) for English language editing.

\section{References}

Arkin, E., Chew, L., Huttenlocher, D., Kedem, K. and Mitchell, J., 1991. An efficiently computable metric for comparing polygonal shapes. IEEE Trans. Pattern Anal. Mach. Intell. 13(3), pp. 209-216.

Dorling, D., 1996. Area Cartograms: Their Use and Creation. School of Environmental Sciences, University of East Anglia, Norwich, England.

Foster, M., 2019. Statistical mapping (enumeration, normalization, classification). In: J. P. Wilson (ed.), The Geographic Information Science \& Technology Body of Knowledge, 2nd quarter 2019 edn, University Consortium for Geographic Information Science, Ithaca.

Gastner, M. T., Seguy, V. and More, P., 2018. Fast flow-based algorithm for creating density-equalizing map projections. Proc. Natl. Acad. Sci. U.S.A. 115(10), pp. E2156-E2164.

Olson, J. M., 1976. Noncontiguous area cartograms. Prof. Geogr. 28(4), pp. 371-380.

Palache, A., Oriol-Mathieu, V., Fino, M. and Xydia-Charmanta, M., 2015. Seasonal influenza vaccine dose distribution in 195 countries (2004-2013): Little progress in estimated global vaccination coverage. Vaccine 33(42), pp. 5598-5605.

Tingsheng, S., Duncan, I. K. and Gastner, M. T., 2019. go-cart.io: A web application for generating contiguous cartograms. Abstr. Int. Cartogr. Assoc. 1, pp. 333. 